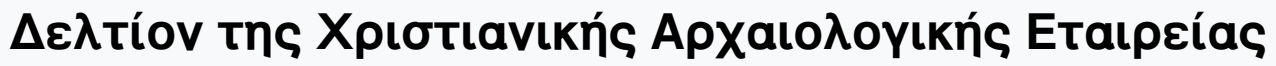

Tóp. 4 (1966)

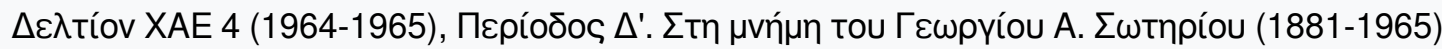

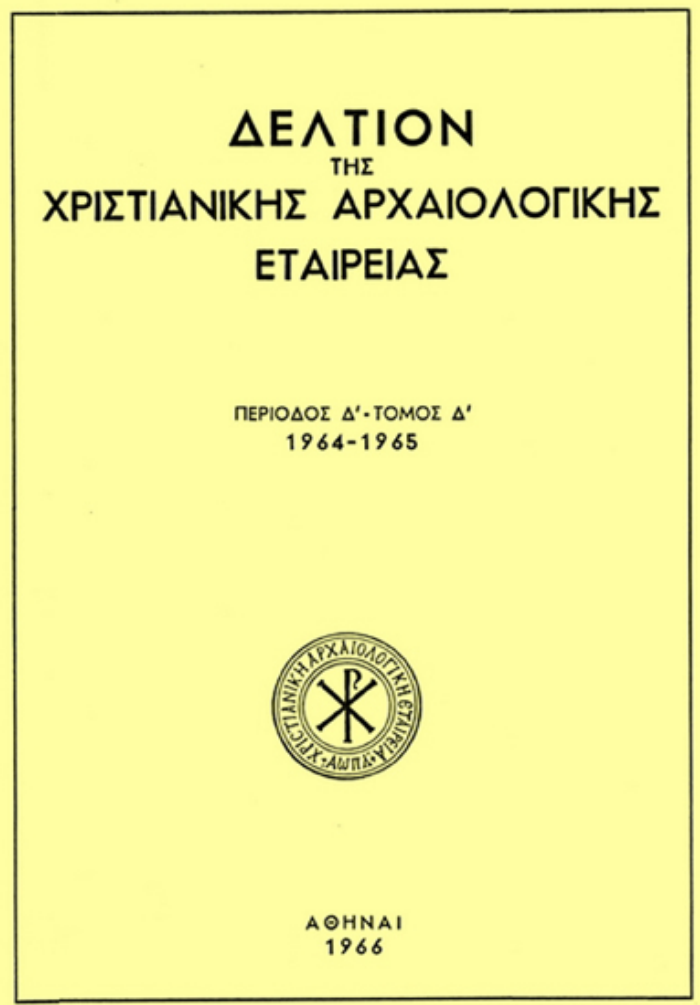

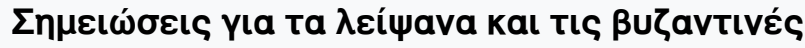

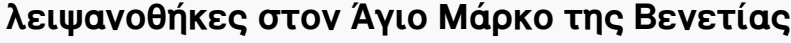

Anatole FROLOW

doi: $\underline{10.12681 / \text { dchae. } 763}$ 


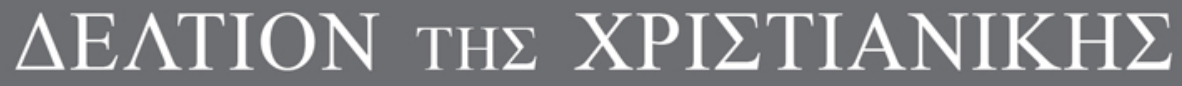 APXAIO $\Lambda$ OГIKH $\Sigma$ ETAIPEIA $\Sigma$}

Notes sur les reliques et les reliquaires byzantins de Saint-Marc de Venise

Anatole FROLOW

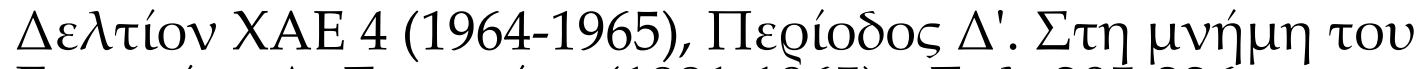

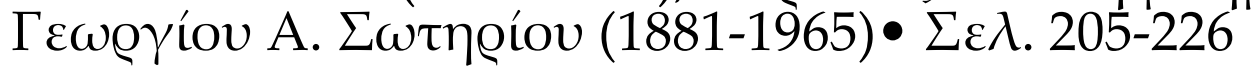

A@HNA 1966 


\section{NOTES SUR LES RELIQUES ET LES RELIQUAIRES BYZANTINS DE SAINT-MARC DE VENISE}

1. Le trésor d'une église est un ensemble mouvant, susceptible aussi bien de diminuer que d'augmenter, suivant les circonstances. Le zèle des donateurs peut avoir des effets intempestifs. Transferts et aliénations sont toujours possibles, sinon souhaités. On ne saurait comparer l'inventaire d'une sacristie à un catalogue de musée, en principe ne varietur, sauf en ce qui concerne les acquisitions nouvelles et éventuellement les dates et les attributions.

L'observation est suggérée par l'examen des reliquaires byzantins du trésor de Saint-Marc de Venise. Il s'agit de quatorze ou quinze objets, soit d'une fraction relativement peu importante des quelques cent-dix reliquaires qui appartiennent à la basilique. Pourtant, le prestige de Byzance s'ajoutant à la valeur cultuelle, l'attention se porte de préférence sur cette petite série de monuments. Pour s'assurer des changements qui sont intervenus dans leur conservation au cours de l'époque la plus récente seulement, il suffira de comparer la monographie bien connue d'Antonio Pasini, Il Tesoro di San Marco in Venezia (Venise, 1887) à la liste des reliquaires découverts en avril 1617, d'une façon qui sembla providentielle, dans la maçonnerie du sanctuaire de Saint-Marc, et dont le futur patriarche Giovanni Thiepolo, alors Primicier de la Procurature, publia aussitôt une description sous la forme d'une plaquette intitulée Trattato della Sanctissime Reliquie ultimamente ritrovate nel Santuario della Chiesa di San Marco (Venise, 1617).

Parmi les objets décrits par Thiepolo et qui n'existaient plus au temps où Pasini rédigeait sa monographie, l'un des plus intéressants semble avoir été une staurothèque en argent doré et repoussé 'en forme de tableau représentant Constantin et Hélène et les bustes des archanges Michel et Gabriel. La relique de la Vraie Croix enchâssée entre ces divers personnages était accompagnée d'une dédicace rédigee au nom d'un dignitaire byzantin, le patrice et triérarche Constantin que le R.P. Vitalien Laurent me propose d'identifier avec Constantin Lips, ce qui permettrait de dater l'objet du début du $\mathrm{X}^{\mathrm{e}}$ siècle: 


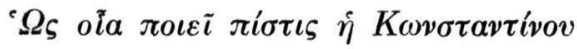

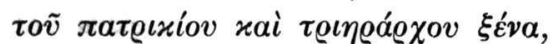

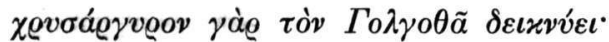

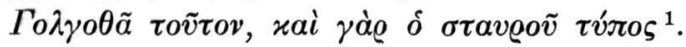

Pasini transcrit, d'après Thiepolo, la traduction latine de ce texte et ajoute "questo prezioso reliquiario.. non esiste più, e s'ignora come e quando sia scomparso" ${ }^{2}$.

Dans d'autres cas, la relique demeure mais son enveloppe a été remaniée ou remplacée par un objet de provenance différente: «alcune reliquie sono custodite in reliquarî ben diversi dai primitivi» ${ }^{3}$. On ne saurait identifier la monstrance anépigraphe en cristal contenant deux Epines de la Sainte Couronne qui a été décrite par Pasini ${ }^{4}$ avec le reli-

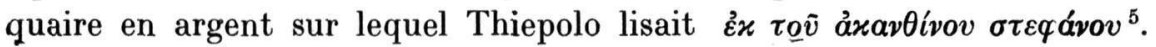
La relique d'un os de saint Pantélémon accompagnée de la légende $\tau o \tilde{v}$

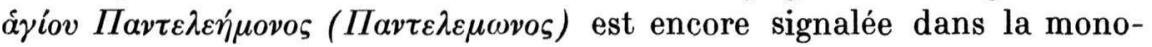
graphie de Pasini ${ }^{6}$, mais il n'y est pas question d'un autre reliquaire contenant du sang du même saint ${ }^{7}$, tandis que la châsse du bras de saint Pantélémon avait déjà perdu à l'époque où cet ouvrage a été rédigé l'inscription métrique qui en proclamait les vertus curatives :

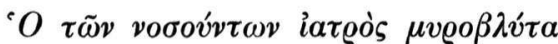

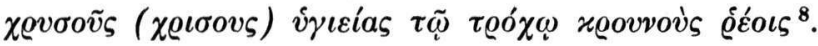

Le trésor de Saint-Marc conserve encore aujourd'hui un os du bras de saint Georges revêtu d'une mince lame d'argent ornée de deux trimètres iambiques :

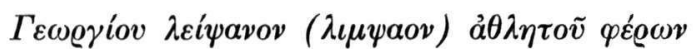

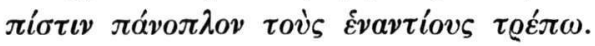

1. Th i e pol o, Trattato, pp. 51 sq. et 69 . Texte grec de la dédicace: CIG, IV ( $\mathrm{K}$ i r c h h off ), p. 371, no 8812, d'après $\mathrm{Mel}$ e t i o s, archévêque d'Athènes,

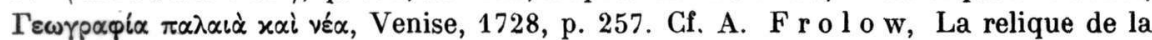
Vraie Croix, Recherches sur le développement d'un culte, Paris, 1961, p. 425, no 528.

2. P a s in i, Il Tesoro, p. 12.

3. Ilidem, p. 23.

4. Ibidem, p. 31.

5. Thie rolo, Trattato, p. 73. L'inscription a été signalée encore en 1860 par J. D u r a n c., L'art byzantin à Saint Marc de Venise, Annales Archéologiques, $\mathrm{XX}$, p. 313.

6. P a s i n i, Il Tesoro, p. 38.

7. Thiepolo, Trattato, p. 87.

8. Trattato, ibiden; P a s i n i, Il Tesoro, p. 36. 
L'objet a été décrit aussi bien par Thiepolo que par Pasini ${ }^{1}$, mais seul le premier de ces auteurs signale deux autres reliquaires avec des légendes grecques désignant les cendres du même saint, réunies, dans l'un des deux cas, aux cendres de saint Théodore ${ }^{2}$. Enfin, c'est seulement grâce à Thiepolo que nous sommes renseignés sur l'existence d'authentiques rédigées en grec qui accompagnaient les reliques de plusieurs autres saints déposées dans un coffret en argent divisé en quinze compartiments ${ }^{3}$. La description de Pasini fait état de légendes latines qui ne coincident pas entièrement, du reste, avec les textes copiés par Thiepolo. Le nom d'Achilleus est devenu Ecaïus, celui de Martinianos a été transformé en Martin, celui de Panthérios de Lampsaque en Partomius, enfin la désignation d'une nouvelle relique est venue s'y ajouter: celle de saint Anélodiste (Anempodiste?) 4 .

Le trésor de Saint-Marc a donc subi des transformations et a même pu s'appauvrir en une certaine mesure entre le début du XVIIe siècle et la fin du XIXe siècle. Les causes particulières qui ont contribué à ce résultat nous échappent. Il est possible que telle est la destinée de tous les trésors ecclésiastiques qui, pour être jalousement gardés, n'en sont pas moins ouverts aux initiatives individuelles de ceux qui en assument la responsabilité ${ }^{5}$. En tout état de cause, dans ce cas précis, le fait demeure patent. Il a semblé opportun de le mettre en lumière.

2. Que savons-nous de la provenance exacte des reliquaires byzantins de Saint-Marc? Une importance sans doute excessive a été attribuée à cet égard aux apports de la Quatrième croisade. Suivant Emile Molinier, les neuf dixièmes du trésor de la basilique marcienne n'avaient d'autres source que le sac de Constantinople en $1204^{6}$. L'affirmation demanderait à être contrôlée pour les émaux et les vases liturgiques les plus précieux. Il est plus difficile encore de la soutenir à propos d'objets dont la valeur est surtout d'ordre cultuel.

1. Th i e p o lo, Trattato, p. 88; P a s in i, Il Tesoro, p. 44. Les descriptions des deux auteurs coincident également en ce qui concerne les reliques de saint Christophe et de sainte Marthe.

2. Thi e p o l o, Trattato, p. 88.

3. Ibidem, p. 84 sq.

4. P a s in i, Il Tesoro, p. 85.

5. Cf. E. L e s n e, Histoire de la propriété ecclésiastique en France, III, L’inventaire de la propriété, Eglises et trésors des églises, Lille, 1936, p. $161 \mathrm{sq}$.

6. E. M o li n i e r, Le trésor de la basilique de Saint Marc à Venise, Venise, 1888, p. 25. Cf. P a s in i, Il Tesoro, p. 23. 
Une simple constatation peut suffire à mettre en garde, dès le premier abord. Les églises de Venise possèdent plus d'une relique apportée de l'Orient avant le XIII' siècle. Le doge Domenico Michiel avait acquis en 1125 un fragment de la colonne de la Flagellation ${ }^{\mathbf{1}}$, le corps de saint Marc avait été transféré en $828^{2}$, celui de saint Tarasius entre 1018 et $1025^{3}$, celui de saint Etienne en $1100^{4}$, celui de saint Nicolas en $1102^{5}$.

D'autres apports, plus récents, sont soit étrangers au pillage de 1204, soit postérieurs à 1261 lorsque Constantinople cessa d'être latine. Il en est ainsi du corps de saint Théodore Tiron envoyé par Jacques Doria, en 1267, de Mésembria sur la Mer Noire ${ }^{6}$ et de la chaire de saint Marc transportée de Grado quelques années seulement avant $1534^{\top}$. La relique de la base de la colonne de la Flagellation fut déposée au trésor de Saint-Marc en 1559: elle provenait de Jérusalem ${ }^{8}$. Le reliquaire des saints de Trébizonde qui appartient également au trésor de la basilique ${ }^{9}$, a dû être exécuté au XV-e siècle, dans l'Empire indépendant fondé par les Comnènes sur le Pont. Le style de cet objet et les caractères paléographiques des inscriptions avec de nombreuses lettres liées qui l'accompagnent

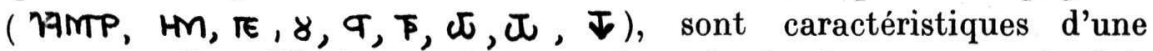
époque tardive. C'était peut-être un présent du dernier empereur de Trébizonde, David Comnène, qui entretenait des relations suivies avec les

1. P a s in i, Il Tesoro, p. 31 .

2. Andrea D a ndolo, Chronicon Venetum, VIII, 2, 5-6 (L. M u rat $0 \mathrm{ri}$, Rerum italicarum scriptores, Milan, 1723 sq., XII, p. 170 sq.); AA. SS., avril, III, p. 352 sq.

3. D a n d o lo, Chronicon, IX, 2, 8 ( M u r a to ri, XII, p. 236 sq.).

4. F. Cornelius (Cornaro), Ecclesiae Venetiae antiquis monumentis... illustratae, Venise, 1749 sq., VIII, p. 96 sq.

5. Historia de translatione sanctorum..., XL, Recueil des Historiens des Croisades, Hist. occ., V, p. 280.

6. C o r n e li u s, op. cit., II, p. 258 sq.: texte de la Translatio reproduit par P. R i a n t, Exuviae sacrae Constantinopolitanae, Genève, 1876 sq., I, p. 156 sq., cf. ibidem, II, pp. 261, 263 et 266 les extraits correspondants des chroniques de Andrea Dandolo et de Marino Sanudo le Jeune.

7. DACL, III, 1, col. 51, s.v. "Chaire épiscopale».

8. P a s i n i, Il Tesoro, p. 39.

9. Ibidem, p. 84 sq., pl. LXIV, 156-8; T h i e p ol o, Trattato, p. 89 sq., E. Molinier (Trésor, p. 102) et G. Schlumberger (L'Epopée byzantine à la fin du Xe siècle, Paris, 1896 sq., I, p. 669, II, pp. 456, 469, 484 et 504) ont certainement trop vieilli cet objet en l'attribuant au XIe ou au XIIe siècle. Voir aussi J. B ra u n, Die Reliquiare des christlichen Kultes und ihre Entwicklung, Freiburg I./B., 1940, pl. 20, fig. 66 . 
puissances de l'Occident dont il sollicitait le secours ${ }^{1}$. Ou bien ce pouvait être un legs du cardinal Bessarion, originaire de Trébizonde, qui avait laissé à Venise, en même temps que sa bibliothèque, diverses reliques destinées à la confrérie de la Charité ${ }^{2}$, mais dont l'une au moins, - celle du vêtement de pourpre du Christ, - a été transportée, plus tard, à Saint-Marc ${ }^{3}$. On tiendra compte aussi des apports possibles des nombreuses familles venues s'installer dès le début du XVe siècle, avec l'assentiment de Venise, de la Mer Noire en Crète ${ }^{4}$. La relique des dents de saint Tite de Gortyne ${ }^{5}$, une poignée de terre imprégnée du sang du Christ ${ }^{6}$ furent, en tous cas, envoyées à Saint-Marc après la prise de l'île par les Turcs, en 1669. Même les Musulmans pouvaient contribuer à certains de ces transferts. Ainsi d'une staurothèque, aujourd'hui perdue, que le pasha de Bosnie Chusocheg avait adressée, en 1528, au doge Andrea Gritti ${ }^{7}$. Il est vrai que, dans ce cas, le reliquaire était marqué aux armes pontificales. Les rapports avec Constantinople et la Quatrième croisade paraissent d'autant plus incertains ${ }^{8}$.

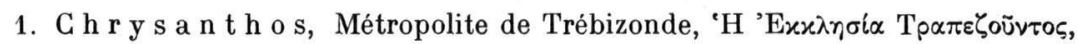
Athènes, 1936, pp. 367 et 534 .

2. Voir pour ces legs: A. V a s t, Le cardinal Bessarion, Etude sur la chrétienté et la Renaissance, Paris, 1878 , p. 425 sq. et $\mathrm{Ch}$ r y s a $\mathrm{nth}$ os, op. cit., p. 300.

3. P a s in i, Il Tesoro, p. 32 sq., pl. XXV, 30.

4. N. N o i $\mathbf{r}$ e t, Documents inédits pour servir à l'histoire de la domination vénitienne en Crète, Paris, 1896, pp. 72 sq. et 225. Pour les rapports amicaux entretenus, d'une façon générale, par l'Empire de Trébizonde avec Venise, voir W. M i lle r, Trebizond, The Last Greek Empire, Londres, 1926, p. 94.

5. P a s in i, Il Tesoro, pp. 36,39 et $46, \mathrm{pl}$. XXXIII, 57.

6. Ibidem, p. 26, pl. XXXVIII, 35.

7. Ibidem, p. 10; F r ol o w, La Relique, p. 618, no 1002.

8. Cf. des exemples analogues, en dehors des reliquaires: une coupe en turquoise offerte à Venise, en 1472, par le shah de Perse Uzun Hasan ( P a s i n i, Il Tesoro, p. 94 sq., pl. XLVIII, 105) et une broderie byzantine du XIIIe siècle envoyée non pas de Constantinople mais du despotat indépendant de l'Epire, dont le fondateur s'était engagé, en 1210, à fournir annuellement un précieux tissu d'or à Saint-Marc et,

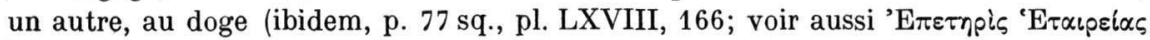

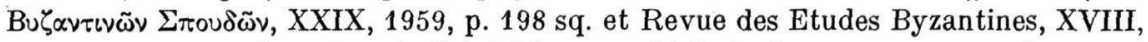
1960, p. 208 sq.). Le trésor de Saint-Marc possédait également une corne de narval (= «licorne»), - aujourd'hui perdue, - cerclée d'un anneau qui portait le nom de l'empereur Jean Paléologue. Suivant Pasini, c'était Jean VIII (op. cit., p. 91, no 2). On pourrait songer aussi bien à Jean V qui avait engagé à Venise, en 1343, un lot d'objets précieux pour une somme de 30.000 ducats ( F. Th ir i e t, Regestes des délibérations du Sénat de Venise concernant la Romanie, Paris - La Haye, I, 1958, no 157; en 1368, le prêt n'était toujours pas remboursé et il était question de vendre les gages: ibidem, no 459, cf. nos 195, 210 et 275). 
Dès lors, on ne saurait affirmer que toutes les reliques orientales conservées à Saint-Marc provenaient nécessairement du sac de la cité impériale en 1204. La réserve est d'autant plus justifiée que le chapitre de Saint-Marc était loin d'être le bénéficiaire exclusif de la générosité des conquérants. L'abbaye de Saint-Georges le Majeur avait reçu les corps de sainte Lucie et de saint Eutychius ${ }^{1}$, le monastère de Saint-Daniel celui de saint Jean d'Alexandrie ${ }^{2}$, l'église Saint-Julien celui de Paul, martyr de l'époque iconoclaste ${ }^{3}$, la chapelle de la Sainte-Trinité celui d'Anastase le Moine ${ }^{4}$. Ont été également déposées en dehors de SaintMarc, les reliques du vieillard Siméon, de Jean de Césarée, de Paul l'Hermite, d'Anastase le Perse et des saintes Hélène, Marine et Barbe ${ }^{5}$. Les donateurs pouvaient être des participants plus ou moins obscurs de la croisade: Valaresus de Valaresiis pour le corps d'Anastase le Moine, Andrea Baldovino et Angelo Drusiaco pour les reliques du vieillard Siméon qui échurent à une église placée sous le vocable de ce saint, un certain Raphael pour les reliques de sainte Barbe qui vinrent enrichir l'église Sainte-Marie-des-Croisés. Giovanni di Bora avait donné à San Liberale le corps de sainte Marine et Jacopo Lantzlo celui de Paul l'Hermite à l'église San Giuliano. Longue liste de noms que vient clore celui du chef de l'expédition lui-même. Les bénédictins de Saint-Georges le Majeur tenaient, en effet, du doge Henri Dandolo le corps de sainte Lucie. La basilique marcienne n'était donc pas le lieu de dépôt unique des apports des croisés.

En fait, les textes, - en l'occurence les chroniques de Venise, toutes postérieures, du reste, au milieu du XIVe siècle, - ne mentionnent qu'un seul envoi adressé à Saint-Marc. Le lot était composé de quatre pièces seulement: une staurothèque gemmée en or, - la croix de Constantin le Grand, disait-on, - une relique du sang du Christ, un bras de saint Georges et un fragment du chef de saint Jean-Baptiste. L'expéditeur était Henri Dandolo ${ }^{6}$. Un simple chevalier allemand, Henri von Uelmen, avait été mieux servi en emportant dans ses bagages un chargement autrement considérable de choses saintes destinées à diverses églises de la Rhénanie ${ }^{7}$. La république de Venise, dont le rôle fut prépondérant au

1. $\mathrm{R}$ i a $\mathrm{n}$ t, Exuviae, II, pp. 262 sq. et 272 sq.

2. Ibidem, I, p. 179 sq.; II, pp. 263 sq. et 294.

3. Ibidem, I, p. 186 sq.; II, pp. 37 sq. et 263.

4. Ibidem, II, p. 261.

5. Ibidem, I, p. 182 sq.; II, pp. 37 sq., 262 sq. et 272 sq.

6. Ibidem, II, pp. 262 sq. et 265.

7. F r o l ow, La Relique, p. 399, no 476. 
début de l'expédition, semble avoir été en perte de vitesse au moment du partage du butin. Le fait est attesté en ce qui concerne les possessions territoriales, la part destinée aux Vénitiens ayant été considérablement réduite après la victoire, au bénéfice des autres croisés ${ }^{1}$. Il a dû en être de même dans le partage des dépouilles sacrées de Constantinople.

3. Parmi les choses saintes adressées à Venise par Henri Dandolo, seule la relique du bras de saint Georges peut être identifiée avec certitude. Les trois autres pièces de l'envoie peuvent correspondre, chacune, à plusieurs reliques de la même nature, ce qui engage à poser la question de l'origine exacte des reliquaires qui les enchâssent. La constatation ne surprend pas en ce qui concerne la Vraie Croix et les cendres de saint Jean-Baptiste que la dévotion des fidèles a contribué de tout temps à multiplier, aussi bien l'une que les autres. Suivant saint Paulin de Nole, on pouvait détacher sans cesse des parcelles du bois imprégné du sang du Christ, sans que le volume en diminuât pour autant ${ }^{2}$. De son côté, Guibert de Nogent s'était montré inquiet de voir le chef du Précurseur conservé simultanément en deux endroits différents ${ }^{3}$. Pour ce qui est de la relique du sang du Christ, la comparaison s'imposerait plutôt avec le phénomène du "dédoublement» des saints fréquemment observé dans la littérature hagiographique ${ }^{4}$. Dans un cas comme dans l'autre, nous ne saurions le plus souvent distinguer qu'à titre d'hypothèse les offrandes de Henri Dandolo des autres objets analogues parvenus au trésor de Saint-Marc par des voies différentes.

C'est bien l'absence de tout pendant qui écarte la discussion sur la provenance du bras de saint Georges ${ }^{5}$. La relique a été déposée dans une châsse émaillée de travail vénitien du XIII ${ }^{\mathrm{e}}$ siècle, cependant l'origine en est confirmée par une mince lame en argent qui l'enrobe, à l'intérieur

1. N. S o k o l o v, La part de Venise dans l'(héritage» byzantin, Vizantijskij Vremennik, N.S., VI, 1953, p. 156 (en russe).

2. P a u l in D e Nole, Epistolae, XXXI, 6, éd. H a r t e l, CSEL, XXIX, p. 274. Cf. F r olow, La Relique, pp. 50 et 169.

3. Guibert D e $\mathrm{N}$ oge nt, Gesta Dei per Francos, 1, 5, Migne, PL., CLVI, col. 695 et De pignoribus sanctorum, 1, 3, ibidem, col. 624. Cf. A. L e f r a n c, Le Traité des reliques de Guibert de Nogent et les commencements de la critique historique, Etudes d'Histoire du Moyen Age dédiées à G. Monod, Paris, 1896, pp. 289 et 303 .

4. Cf. H. D e l e h a y e, Les légendes grecques des saints militaires, Paris, 1909, p. 15 sq.

5. P a s i n i, Il Tesoro, p. 43 sq., pl. XXX, 45; cf. ci-dessus p. 207, n. 1. 
de ce reliquaire, et qui porte, pour tout ornement, une inscription grecque en deux lignes dont chacune forme un dodécasyllabe iambique : nous venons d'en reproduire ci-dessus le texte.

La relique du chef de saint Jean-Baptiste n'est représentée, elle aussi, que par un seul exemplaire; toutefois il y a lieu de tenir compte, dans ce cas, de deux châsses différentes. La question se pose en raison d'un incendie qui s'était déclaré en 1230, dans le sanctuaire de SaintMarc ${ }^{1}$. Le reliquaire du chef du Précurseur figurait parmi les objets endommagés par les flammes mais le contenu en fut épargné et on a pu le transporter dans un nouveau récipient. Actuellement la relique est déposée dans un calice byzantin exécuté aux frais du proèdre Basile, bâtard de l'empereur Romain I-er Lécapène ${ }^{2}$. Une notice de l'inventaire dressé en 1325 laisse entendre qu'un autre vase, plus approprié, était prévu à l'origine: Item de capite Snci Johannis Baptistae in quadam capsicula Argenta deaurata cum literis de nielo circundata et cum figuris in cohopertura ${ }^{3}$. Suivant Pasini, l'objet est conservé encore aujourd'hui dans le trésor de la basilique. Il s'agirait d'une petite boîte en argent avec un couvercle à coulisse orné d'une image de saint Jean-Baptiste debout, tenant à la main un phylactère déployé sur le verset de Matthieu, IV,

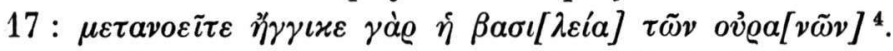

L'identification est douteuse. Dans le texte de l'inventaire de 1325, il est question de figures au pluriel et d'une inscription niellée dans le pourtour, ce qui n'est pas le cas du petit écrin que l'on vient de décrire. A en juger d'après le style, l'œuvre ne semble pas, du reste, être antérieure au XVe ou au XVIe siècle. Sous le rapport de l'iconographie, il y a lieu en particulier de tenir compte du coussin placé sous les pieds du Précurseur. Dans l'art byzantin, ce détail se rencontre moins souvent dans l'iconographie des saints que dans les portraits des empereurs ${ }^{5}$. Un exemple analogue, parmi les plus anciens, est offert par un panneau

1. R i a n t, Exuviae, II, p. 149 sq.

2. P a s i n i, Il Tesoro, p. 42, pl. XXXIII, 50. Cf. Ch. D i e h l, De la signification du titre de (proèdre» à Byzance, Mélanges... G. Schlumberger, Paris, 1924, I, p. 110.

3. P a s i n i, Il Tesoro, Appendice, I, p. A 4.

4. Ibidem, p. 43, pl. XXIX, 42. Cf. M ol i n i e r, Trésor, p. 87, qui attribue l'objet, d'une façon plus affirmative, au XIII' siècle.

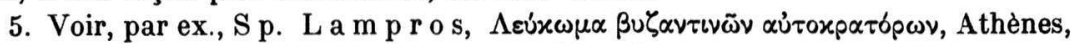
1930, pl. 65 (Alexis Ier Comnène), 69 (Manuel Ier Comnène et sa femme Marie), 73 (Théodore II Lascaris), 75 (Michel VIII Paléologue), etc. 
peint de l'école byzantinisante de Pise qui représente l'archange Michel ${ }^{1}$. Il paraît raisonnable d'admettre que la capsicula décrite dans l'inventaire de 1325 n'existe plus. La petite boite qui nous est parvenue doit être postérieure à cette date: on en ferait volontiers honneur à un orfèvre grec établi en Italie. Nul doute cependant que le contenu en fut constitué par des cendres du Précurseur. Le culte de ce saint était à coup sûr assez répandu pour que deux de ses reliquaires vinssent successivement enrichir le même trésor.

L'identification de la croix offerte par Henri Dandolo pose un problème plus difficile à résoudre, dans la mesure où Saint-Marc possède plusieurs staurothèques de l'époque byzantine. Le choix de Pasini se porte, dans ce cas, sur un reliquaire qui, de même que celui du chef de saint Jean-Baptiste, fut endommagé dans l'incendie de 1230. Une inscription gravée au revers de l'objet précise qu'il a été refait en 1517. Les restaurateurs ont toutefois pris soin de respecter, dans la mesure de leurs moyens, l'aspect de l'original disparu. L'iconographie, - Constantin et Hélène autour d'une croix à double traverse, - est bien celle de la plupart des autres staurothèques byzantines, et l'on a même reproduit la dédicace qui désignait en qualité de donatrice une impératrice Maria, peut-être Marie d'Allanie, femme de Nicéphore III Botaneiatès, ou Marie d'Antioche, femme de Manuel Ier Comnène :

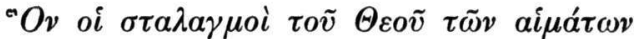

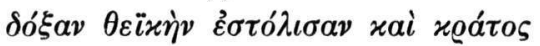

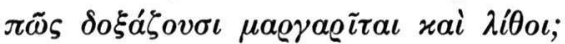

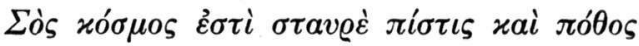

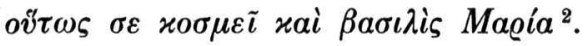

Un inventaire de Saint-Marc dressé en 1283 viendrait à l'appui de l'identification de Pasini. Le texte ne mentionne, en effet, qu'une seule

1. W. Felicetti - Li ebenfels, Geschichte der byzantinischen Ikonenmalerei, Olten-Lausanne, 1956, pl. $98 \mathrm{~B}$. Un rapprochement peut être également proposé avec les icônes de l'époque post-byzantine où l'on voit un coussin posé sur le marchepied du trône du Christ ou de la Vierge: par ex. A. X y n g o p o ul o s,

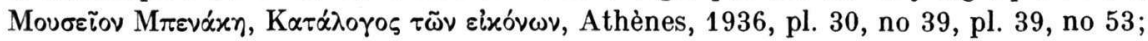
V. D j u r i ć, Icônes de Yougoslavie, Beograd, 1961, pl. LXVIII, no 48; M. C h at z i d a k i s, Icônes de Saint-Georges des Grecs..., Venise, 1963, pl. 6, 8 et 55.

2. P a s in i, Il Tesoro, p. 27 sq., pl. XXVI, 33; Frolow, La Relique, p. 296 sq. no 273 (bibliographie). 
staurothèque, - celle précisément de l'impératrice Marie ${ }^{1}$. Ceci peut laisser entendre que vers la fin du XIIIe siècle le trésor n'en possédait encore aucune autre. Le manuscrit est toutefois incomplet, si bien qu'il eut été plus hasardeux que jamais de vouloir tirer ici parti de l'argument $a b$ silentio. Une tradition différente de celle de Pasini a été, du reste, attestée par Francesco Sansovino, qui écrivait au XVI-e siècle. Cet auteur distingue entre la staurothèque endommagée en 1230 et la "croix de Constantin" ${ }^{2}$, or c'est ainsi que d'autres sources anciennes désignent, nous l'avons déjà rappelé, - le reliquaire envoyé par Henri Dandolo.

Le plus sûr est de procéder par élimination. Citons, surtout pour mémoire, une petite boîte en argent doré munie d'un anneau de suspension et qui contient, dans une cavité cruciforme ménagée au milieu de la face antérieure, deux morceaux de bois censés provenir de la Vraie Croix ${ }^{3}$. Tout autour, dans les coins de la boîte, se détachent des têtes de clous qui devaient maintenir un revêtement métallique: on imaginera une ouverture ajourée, en forme de croix également, de façon à laisser apercevoir la relique. Il est possible qu'il faille reconnaître la description de cet objet dans une notice de l'inventaire de 1325: Capsiculetam I. parvam quadratam argenti in qua est de ligno Domini ${ }^{4}$. L'identification fournirait un terminus ante quem intéressant. Il est plus difficile de supposer que ce soit un apport de la Croisade. Nous aurons l'occasion de citer ci-dessous des staurothèques grecques qui enchâssent un morceau de la relique insigne taillée en forme de simple croix. Ce sont cependant des exceptions. Le plus souvent, on est en présence de croix à double traverse qui tiennent une place relativement plus importante dans l'économie du décor. Le reliquaire de Saint-Marc serait plutôt à comparer, à cet égard, à la plupart des staurothèques d'origine occidentale ${ }^{5}$. En tout état de cause, il paraît impossible de reconnaître dans ce modeste encolpion, qui ne mesure guère plus de $10 \mathrm{~cm} . \times 3 \mathrm{~cm}$., la mirifica crux auro inclusam dont il est question dans les textes relatifs à l'envoie de Henri Dandolo.

1. P a s in i, Il Tesoro, Appendice, I, p. A 3. La notice est précédée de points de suspension "Deinde....), puis vient la description de la staurothèque endommagée dans l'incendie de 1230 : Item crux Christi qui fuit in igne in una ycona...

2. R i a $\mathrm{t}$, Exuviae, II, p. 268.

3. P a s in i, Il Tesoro, p. 39.

4. Ibidem, Appendice, I, p. B 4.

5. Par ex., F r o low, La Relique, pp. 314, 354, 356, 464, nos 306, 393, a et b, 395, 612, etc. 
Même difficulté en ce qui concerne un reliquaire perdu de la Vraie Croix qui devait être décoré, à l'origine, de nielles et d'émaux cloisonnés et détourés qu'un orfèvre vénitien du XIIIe ou du XIVe siècle avait transporté, du moins en partie, sur un panneau en argent doré destiné à servir soit d'icône, soit de plat de reliure ${ }^{1}$. L'objet est formé de onze appliques en émail qui représentent la scène du Crucifiement; une lame niellée avec une inscription métrique incomplète ou mal rédigée s'y ajoute, précisant que l'ensemble provenait d'une staurothèque :

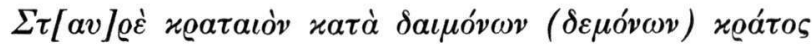

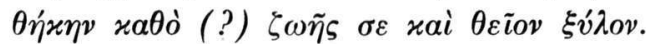

Le cadre du panneau est décoré de deux médaillons émaillés avec des bustes d'anges qui ont du être détachés de quelque autre pièce du mobilier liturgique. La facture en est beaucoup plus délicate que celle des appliques avec la scène du Crucifiement, ce qui implique une différence de date. Les médaillons peuvent être attribués, sous toute réserve, au $\mathrm{X}^{\mathrm{e}}$ ou au XIe siècle, les appliques au XII $\mathrm{X}^{\mathrm{e}}$. Il n'est donc pas exclu au point de vue chronologique que le tableau original faisait partie du butin des croisés en 1204. La difficulté réside dans le problème de la provenance. Les émaux de style byzantin à la fois cloisonnés et détourés sont relativement rares; aucun ne peut être attribué avec certitude à un atelier de Constantinople. L'exemple le plus approchant qu'il y a lieu de rappeler ici est offert par la grande figure du Christ au Musée Kircher à Rome, qui date peut-être au début du XIIIe siècle et dont il semble qu'il faille faire honneur à un artiste italien ${ }^{2}$, ainsi qu'il en est des émaux de la cathédrale de Capoue ${ }^{3}$ ou encore de ceux qui ont été ajoutés, en 1209 au plus tôt, à la Pala d'Oro de Saint-Marc. D'autres comparaisons sont à faire avec des émaux sur cuivre, en partie cloisonnés, en partie champlevés, et qu'il eut été facile de détourer comme on l'a fait pour le Christ du Musée Kircher. Telles sont une coupe ortokide du Musée d'Innsbruck (1141-1143) ${ }^{4}$ ou une petite icône de saint Théodore conservée au Musée

1. P a s i n i, Il Tesoro, p. 73, pl. III; F r o l o w, La Relique, p. 298, no 275, add. D. T a l b ot R i c e, Art Byzantin, Paris-Bruxelles, 1959, pl. 138 (date soutenue: début du XIe siècle). graphie).

2. O. D a l t o n, Byzantine Art and Archaeology, Oxford, 1911, p. 510 (biblio-

3. Y. $\mathrm{Hacken} \mathrm{b} \mathrm{roch}$, Italienisches Email des frühen Mittelalters, Bâle, 1938 , p. 98 , fig. $55-56$.

4. M. Van Berchem et J. S trzygowski, Amida, Heidelberg, 1910, fig. 51-53, pl. XXI. 
de l'Ermitage et qui pourrait bien avoir été exécutée, au XII ${ }^{\mathrm{e}}$ ou au XIIIe siècle, dans l'une des provinces orientales de l'Empire byzantin ${ }^{\mathbf{1}}$. Certes, Constantinople qui avait drainé, depuis sa fondation, des richesses provenant des contrées les plus diverses, pouvait offrir des objets d'importation à la convoitise des Croisés. Admettre qu'il en avait été ainsi dans le cas présent, eut été cependant étayer une hypothèse par une autre hypothèse: il vaut mieux y renoncer.

La réserve s'impose aussi, cette fois à cause de la date, pour une autre staurothèque qui était affectée, suivant l'inventaire de 1325, à la cérémonie de l'Adoration de la Croix célébrée annuellement à l'office du Vendredi saint ${ }^{2}$. Il s'agit d'un tableau en argent doré muni d'un couvercle à coulisse avec une serrure à targette ajoutée après coup. La relique insigne est logée à l'intérieur, dans un alvéole en forme de croix à double traverse. Seule la partie extérieure du reliquaire conserve son décor original. Sur le couvercle est fixée une plaque en émail cloisonné qui représente la scène du Crucifiement, avec un Christ au corps très déhanché. L'encadrement est formé d'une double rangée de cabochons et de pierres semi-précieuses, maintenus, les uns et les autres, dans des sertissures à griffes. Six médaillons en émail cloisonné avec des bustes de saints et d'apôtres s'ajoutent à cet alignement à des intervalles réguliers. Les émaux sont d'une facture médiocre et s'écaillent en plus d'un endroit, aussi bien dans la scène du Crucifiement que dans les médaillons. Toutes les autres parties du reliquaire sont travaillées au repoussé. Il suffira, pour notre propos, de signaler les bustes de saints, - cinq grands évêques et cinq saints militaires, - qui décorent la tranche.

La forme et les dispositions générales de ce reliquaire se retrouvent dans un groupe de monuments dont le plus ancien, - la célèbre staurothèque de Limbourg-sur-la-Lahn, - date du premier quart du $\mathrm{X}^{\mathrm{e}}$ siècle ${ }^{3}$. Toutefois, dans le cas présent, plusieurs détails sollicitent en faveur d'une époque postérieure à la Quatrième croisade. Les monuments

1. D a 1 t o n, op. cit., p. 528 (bibliographie. Add. Cahiers archéologiques, I, 1945, pl. XXIV, 2 et A. B a n k, L'art de Byzance dans la collection de l'Ermitage (en russe), Léningrad, 1960, pl. 84).

2. P a s in i, Il Tesoro, p. 33 sq., pl XXXIII (date soutenue: IXe siècle); M o li n i e r, Trésor, p. 84 (date soutenue: XIIe siècle); B r a u n, Reliquiare, pl. 19, fig. 64; D. T a l b o t R i c e, The Leaved Cross, Byzantinoslavica, XI, 1950, pl. II (le revers seul); F r o l o w, La Relique, p. 485, n $^{\circ} 663$.

3. Etude stylistique la plus complète de l'ensemble des staurothèques de ce groupe: A. B a n k, Objets byzantins en argent du XIe-XIIe siècle dans la collection de l'Ermitage, 2, Vizantijskij Vremennik, N.S., XIV, 1958, p. 234 sq. (en russe). 
du temps des Macédoniens et des Comnènes ne font guère connaître de représentations du Christ en croix avec la courbe du corps aussi infléchie ${ }^{1}$. Les saints représentés sur la tranche ont les joues trop bombées et rapprochées du nez, ce qui constitue un trait particulièrement caractéristique de l'art des Paléologues ${ }^{2}$. Une indication dans le même sens est offerte par la monture des cabochons qui sont sertis à griffes, comme c'est le cas, par exemple, sur la croix des Zaccaria au Dôme de Gênes, restaurée entre 1260 et $1283^{3}$. Sur un objet d'une époque plus ancienne, on se serait plutôt attendu à trouver des sertissures en forme de bâtes. Il y a lieu, enfin, de tenir compte de la mauvaise facture des émaux cloisonnés, qui semble correspondre à la décadence de cette technique après la prise de Constantinople par les Croisés ${ }^{4}$. Autant de contre-indications qui interdisent d'attribuer à Henri Dandolo l'envoi de ce reliquaire.

L'identification serait plus aisée à soutenir avec deux autres staurothèques. Nous avons déjà signalé l'une d'elles, aujourd'hui perdue, qui était accompagnée d'une dédicace rédigée au nom du patrice et triérarche Constantin. L'autre, qui avait appartenu à l'impératrice Irène Doukas veuve d'Alexis Ier Comnène, fait toujours partie du trésor de la basilique ${ }^{6}$.

On ne saurait tirer argument, en ce qui concerne le premier reliquaire, de la similitude du nom du donateur avec celui de Constantin le Grand à qui les chroniqueurs vénitiens attribuent la croix envoyée en 1204 . D'autres staurothèques ont reçu la même désignation, comme pour en garantir l'authenticité, et sans doute aussi pour ajouter à la révérence qui leur était dûe ${ }^{6}$. Par contre, la date et la provenance de cet objet, exécuté pour un haut dignitaire de la cour de Constantinople, probablement au début de la période macédonienne, pourraient bien convenir à un envoi de la Quatrième croisade.

1. G. Mill e t, Recherches sur l'iconographie de l'Evangile..., Paris, 1916, p. 409 sq. Le plus ancien exemple cité est une fresque du milieu du XIIIe siècle à Zica, en Serbie.

2. Par ex., l'album des mosaiques de Kariye Camii paru en supplément au t. XI des Izvěstija de l'Institut archéologique russe de Constantinople, 1906, pl. V sq. et LXI sq.

3. G. Schlu m b e rge r, La croix byzantine dite des Zaccaria, Monuments... Piot, II, 1895, p. 131 sq. Voir aussi une croix du XVe ou du XVIe siècle conservée au Protaton du Mont-Athos: N. Kon dakov, Monuments de l'art chrétien à l'Athos, S.-Pétersbourg, 1902, pl. XXIX (en russe).

4. Cf. Cahiers archéologiques, I, 1945, p. 100 sq.

5. P a s i n i, Il Tesoro, p. 28 sq., pl. XXV, 29; F r o l o w, La Relique, p. 315, $\mathrm{n}^{0} 308$.

6. Ibidem, p. 89 . 
La remarque s'applique aussi à la staurothèque d'Irène Doukas qui se présente sous l'aspect d'une croix latine formée de deux fragments de la Vraie Croix de dimensions particulièrement considérables pour une relique de cette espèce $(0 \mathrm{~m} .21 \times 0 \mathrm{~m}$. 14, épaisseur $0 \mathrm{~m} .04)$. Les extrémités du montant et de la traverse sont protégées par de petits étuis en argent doré qui portent une dédicace niellée. Il devait y avoir aussi, pour plus de sûreté, un écrin métallique en forme de tableau ou de triptyque, ainsi que le cas se présente pour la plupart des staurothèques byzantines. Ceci correspondrait à la mention d'une croix en or dans les textes relatifs à l'envoi de Henri Dandolo. Une indication dans le même sens est peutêtre à retenir du volume exceptionnel de la relique que des chevaliers latins ignorants la langue grecque pouvaient attribuer, pour cette unique raison, au plus illustre des souverains de Byzance. Quoi qu'il en soit, la dédicace garantit la date et la provenance '. La croix a été exécutée après que l'impératrice, devenue veuve en 1118, ait pris le voile dans un monastère qu'elle avait fondé elle-même à Constantinople sous le vocable de Notre-Dame Pleine-de-Grâce et où elle devait mourir dans les années qui ont suivi 1126 , peut-être en $1133^{2}$.

S'il fallait désigner le reliquaire adressé à Saint-Marc de la capitale conquise, la préférence se porterait sur l'une de ces deux croix, celle du patrice Constantin et celle d'Irène Doukas. Eventuellement, on pourrait y ajouter la staurothèque de l'impératrice Marie, qui avait retenu l'attention de Pasini. Il suffirait d'écarter la contre-indication de Francesco Sansovino qui écrivait, malgré tout, à une époque relativement récente. Ce serait encore réduire de moitié le choix entre six objets différents. Mais plutôt que de préciser davantage, il est préférable d'avouer notre ignorance.

1. Le texte a été déjà reproduit plusieurs fois, avec de légères variantes, notamment dans les ouvrages cités ci-dessus p. 217, note 5 (bibliographie). En voici une

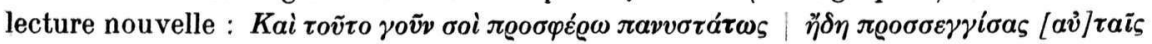

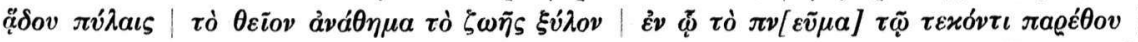

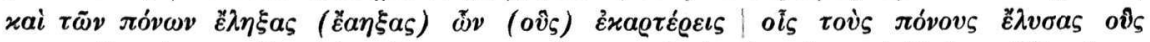

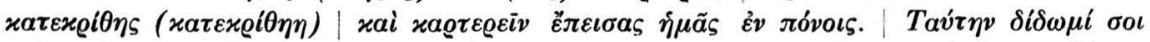

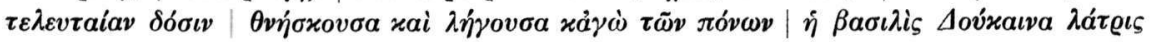

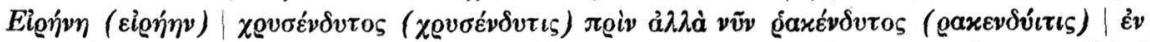

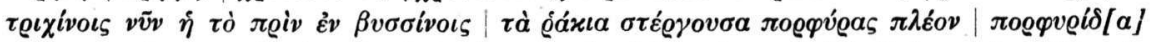

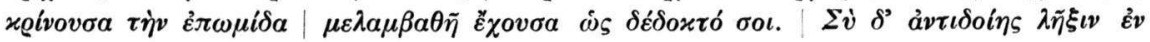

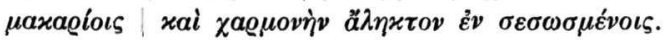

2. F. C h a l a n d o n, Jean II Comnène (1118-1143) et Manuel I Comnène (1143-1180), Paris, 1912, p. 15, n. 2. 
Une difficulté du même ordre s'attache à l'identification de la relique du sang du Christ, encore que les données du problème ne soient pas absolument identiques. Nous avons à tenir compte de cinq reliquaires du sang pour six staurothèques, le nombre n'en est pas moins exceptionnel en raison de la nature de la relique dont l'existence n'a jamais été affirmée avec autant d'insistance en d'autres trésors ecclésiastiques. Pasini a proposé de distinguer, à la sacristie de Saint-Marc, entre la relique du sang du Christ, la relique de la terre imbibée de ce sang et la relique du sang jailli miraculeusement du crucifix de Béryte qu'un Juif avait frappé d'un coup de couteau ${ }^{1}$. Il faudrait admettre une sorte de polarisation de culte exprimé sous des formes diverses mais apparentées les unes aux autres. Le phénomène est assez particulier, et ce sont les légendes hagiographiques où tel saint, par exemple saint Théodore, apparait comme dédoublé, sous des identités différentes, qui semblent apporter ici,-—nous venons de le rappeler, - le terme de comparaison le plus satisfaisant.

Cependant c'est la question de provenance qui importe surtout dans le cas présent. Ainsi que pour les staurothèques, le plus sûr sera de procéder par élimination. Une simple mention peut suffire en ce qui concerne les deux ampoules en cristal, dont l'une a été envoyée, en 1669, de Crête ${ }^{2}$, et l'autre, - offerte en 1630 par le doge Nicolas Contarini à la paroisse de Santa Maria Nuova, - ne fut déposée à Saint-Marc que vers le milieu du XIXe siècle ${ }^{3}$. Un troisième cristal, - celui-ci de facture fatimide, remonte au $\mathrm{XI}^{\mathrm{e}}$ siècle $^{4}$. Ce pouvait être l'envoi d'un Croisé, mais ce n'était pas l'œuvre d'un Grec.

Les deux reliquaires qui restent sont indubitablement d'origine byzantine. C'est, d'abord, un médaillon en or fixé au sommet de la stauro-

1. P a s in i, Il Tesoro, p. 25 pour le sang du crucifix de Béryte, et p. 26 pour la terre imbibée du sang du Christ. L'identification avec le sang du crucifix de Béryte est également soutenue par D u rand, op. cit., p. 309 et, au début du XVII siècle, par Paul Rhamnusius ( $\mathrm{R}$ i a n t, Exuviae, II, p. 270). Les sources plus anciennes sont moins précises. Dans sa description de Venise parue en 1581, Francesco Sansovino hésite entre deux traditions, dont l'une faisait venir la relique de Béryte et l'autre de Constantinople (ibidem, II, p. 267 sq.). Andrea Dandolo et Lorenzo de' Monaci, au XV' siècle, et Marin Sanudo le Jeune, au commencement du $\mathrm{XVI}$ siècle, appellent la relique (miraculeuse" mais rien n'indique que cette épithète ait été choisie par allusion au miracle de Béryte (ibidem, II, pp. 262, 263 et 265).

2. P a s i n i, Il Tesoro, p. 26, pl. XXVIII, 35.

3. Ibidem, p. 27, pl. XXVIII, 37.

4. Ibidem, p. 25 sq., pl. XXIV et XXVIII, 36; C. L a m m, Mittelalterliche Gläser und Steinschnittenarbeiten aus dem Nahen Osten, Berlin, 1929, p. 204, pl. 69. 
thèque de l'impératrice Marie ${ }^{1}$. L'objet est formé de deux volets réunis l'un à l'autre par une charnière. La face antérieure est ciselée et représente le Christ en buste. Sur l'autre face est gravé un vers :

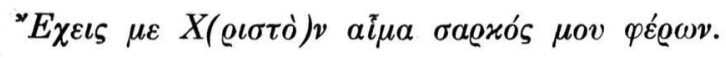

Le travail est d'une finesse exquise. La date de ce petit chef-d'œuvre d'orfèvrerie peut être fixée au XIe siècle. Le buste du Christ sur l'avers rappelle des représentations analogues sur les monnaies frappées entre le règne de Constantin VII Porphyrogénète (944-959) et celui de Michel VII Parapinace (1071-1078) ${ }^{2}$. La ressemblance est particulièrement accusée avec les émissions de Michel IV le Paphlagonien (1034-1041) ${ }^{3}$.

Le second reliquaire dont il y a lieu de tenir compte ici, est constitué par un petit cylindre en cristal de roche qui s'emboîte dans un étui en cuivre doré, également de forme cylindrique, et dont le couvercle (muni d'une serrure à targette) sert de support à un autre récipient (sans serrure), plus petit et bombé comme une coupole en miniature ${ }^{4}$. L'ensemble était apparemment destiné à rappeler un édifice circulaire voûté, analogue aux représentations médiévales du Saint-Sépulcre ${ }^{\mathbf{5}}$.

Deux vers iambiques gravés en capitales accentuées, plus décoratives que régulières, précisent la destination de l'étui métallique :

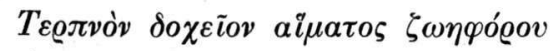

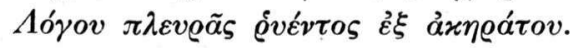

1. Pas in i, Il Tesoro, p. 27.

2. W. Wroth, Catalogue of the Imperial Byzantine Coins in the British Museum, Londres, 1908, pl. LIII, 7 à LXIII, 3. Les représentations du Christ en buste introduites dans la numismatique byzantine par Justinien II, puis reprises sous le règne de Michel III et de Basile Ier, sont d'un type différent.

3. Wroth, op. cit., pl. LVIII, 2. Cf. ibidem, pl. LXI, 9 (Constantin X). La position de la main droite du Christ est la même que sur le reliquaire de Saint-Marc: ce n'est pas le cas des autres émissions analogues.

4. Th i e p o l o, Trattato, p. 46 sq.; P a s i n i, Il Tesoro, p. 24 sq. pl. XXVII et fig. à la p. 89 (dessin du couvercle du cylindre en cristal, d'après Thiepolo; une photographie du même couvercle est reproduite par M. G. R os s, Three Byzantine Cameos, Greek-Roman and Byzantine Studies, III, 1, 1960, pl. 6, fig. 3). Voir aussi D u ra nd, op. cit., p. 309; M o li n i e r, op. cit., p. 86 et Maxime de Syma, O

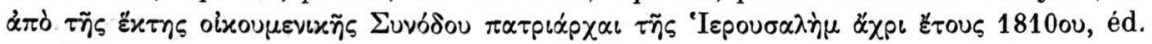

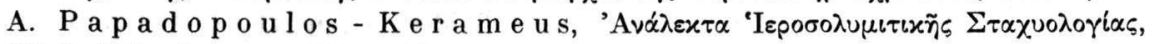
III, S.-Pétersbourg, 1897, p. 29.

5. Cf. E. B a ld w in S m it h, A Source of Medieval Style in France, Art Studies, II, 1924, p. 90, pl. I et A. G r a b a r, Martyrium, Recherches sur le culte des reliques et l'art chrétien antique, Paris, 1946, I, pp. 257 sq. et 270 sq. 
La destination du récipient plus petit soudé au couvercle n'est pas certaine. L'absence de serrure témoignerait qu'en comparaison avec l'étui principal, une importance relativement moindre était attachée à son contenu. Ce pouvait être, à titre d'hypothèse, du baume ou de l'huile sanctifiés, sinon au contact, du moins par la proximité du sang du Christ ${ }^{1}$.

Le bord supérieur du cylindre en cristal, qui sert de reliquaire proprement dit, est cerclé d'une inscription en émail cloisonné. En dehors de plusieurs fautes de iotacisme et d'orthographe, le texte est identique à celui qui est gravé sur le médaillon fixé à la staurothèque de l'impératrice Marie :

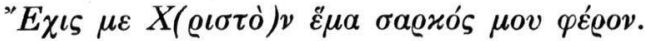

Un couvercle, également décoré d'émaux cloisonnés, vient se rabattre sur cette sertissure. Un crucifix en jaspe vert est encastré en son milieu. Les extrémités de l'intaille échancrent légèrement la bordure décorative qui l'encadre. Il est probable que l'orfèvre a utilisé une applique destinée à un usage différent. Au point de vue de style, il y a des rapprochements à faire avec une intaille de la collection du comte Cini à Venise représentant saint Georges accompagné d'un portrait présumé d'Alexis V Mourzouphle (1204) ${ }^{2}$. L'œuvre serait d'une époque immédiatement antérieure à la Quatrième croisade. La conclusion est confirmée, en une certaine mesure, par la forme des lettres gravées sur l'étui.

Mais comment attribuer à Henri Dandolo, avec le moins de chances d'erreur, l'un ou l'autre de ces deux reliquaires byzantins? Les chroniques relatives à l'envoi du doge n'apportent guère d'indications précises dans ce sens. Les inventaires publiés du trésor de Saint-Marc sont à peine plus explicites. Rien ne peut être tenu, dans ces conditions, pour certain.

Nous ne disposons point, - notons-le tout de suite, - de descriptions anciennes du médaillon fixé à la staurothèque de l'impératrice Marie que l'on attribuerait cependant, le plus volontiers, à un atelier d'orfèvre de Constantinople. La dédicace de la staurothèque où il est question à la fois du bois de la Croix et des "gouttes de sang qui l'ont revêtu de

1. Cf. E. L u c i u s, Les origines du culte des saints dans l'Eglise chrétienne, Paris, 1908, pp. 410 et 440; H. D e l e h a y e, Saints et reliquaires d'Apamée, Analecta Bollandiana, 1935, LIII, p. 237 sq.

2. H. We n t z e l, Datierte und datierbarte byzantinischen Kameen, Festschrift F. Winkler, Berlin, 1959, fig. 2-3; $\mathrm{R}$ o s s, op. cit., p. 45. L'identification avec Alexis Mourzouphle n'est pas absolument certaine, mais il n'est pas douteux que l'intaille soit d'une époque relativement tardive. 
puissance et de gloire «laisserait entendre que les deux reliquaires étaient réunis dès l'origine. L'auteur d'un inventaire pouvait se borner à en signaler un seul, le plus important. Par contre, l'hypothèse ne s'accorde pas avec le témoignage des chroniqueurs qui distinguent, à propos de l'envoi de 1204, entre la relique de la Vraie Croix et la relique du sang du Christ.

Dès lors, le choix se portera de préférence sur l'une des deux ampoules en cristal, même si celle que nous avons décrite en premier lieu n'est pas d'origine byzantine. Pasini désigne sans hésiter le petit cylindre qui s'emboîte dans un étui métallique. L'identification est assurément confirmée par la présence des inscriptions grecques qui font défaut, et pour cause, sur l'ampoule fatimide. L'examen des inventaires qui nous sont accessibles engagerait à plus de réserve. L'inventaire de 1325 mentionne les deux ampoules: ampula de cristallo pour le petit vase façonné par un artiste arabe, busoletus auri in quo est alius busoletus cristali pour le reliquaire byzantin ${ }^{1}$. La première notice figure au début du texte, la seconde occupe seulement la septième place, ce qui eut été malgré tout surprenant si l'auteur avait en vue l'un des envois les plus importants dont le trésor de Saint-Marc s'était enrichi à la suite de la Croisade. D'autre part, dans l'inventaire de 1283 il est question seulement de l'ampoule fatimide, et, là encore, l'objet est cité à la place d'honneur, in primis ${ }^{2}$. A ne tenir compte que de ces indications, il est tentant d'admettre que le reliquaire accompagné de légendes grecques a été acquis par le trésor au plus tôt vers la fin du XIIJe siècle. C'est attribuer à Henri Dandolo l'envoi de l'ampoule fatimide. On serait confronté avec une illustration précise du rôle de Constantinople dans le trafic des œuvres d'art oriental apportées au Moyen Age en Occident. Comment oublier cependant que la démonstration repose essentiellement sur le silence d'un texte et que ce texte est, du reste, incomplet? Une fois de plus, nous sommes amenés à avouer que la solution définitive de ces petits problèmes d'archéologie nous échappe.

4. Si la provenance des reliquaires byzantins de Saint-Marc prête à discussion, leur intérêt n'en demeure pas moins considérable à divers autres égards. Intérêt historique, déterminé par l'identité des premiers donateurs dont le nom évoque les fastes de la cour de Constantinople.

1. P a s i n i, Il Tesoro, Appendice, I, p. A 4.

2. Ibidem, p. A 3 . 
Intérêt archéologique, suggéré par la comparaison de ces objets soit les uns avec les autres, soit avec les reliquaires occidentaux conservés dans le même trésor. C'est l'occasion d'apprécier les ressemblances qu'une destination commune peut déterminer dans une série d'œuvres d'art en apparence la plus disparate. L'opportunité est offerte, en même temps, de confronter les modèles que le prestige de Byzance proposait aux orfèvres occidentaux et le parti que ces orfèvres ont su en tirer pour leurs propres travaux.

En ce qui concerne les similitudes d'ordre fonctionnel, c'est - à - dire celles qui reposent sur la destination des objets, l'attention se portera de préférence sur les trois reliquaires orientaux du sang du Christ, si différents qu'ils soient les uns des autres. Ainsi, tout d'abord, des légendes. Un artiste italien du XIVe siècle a enchâssé l'ampoule d'origine fatimide dans un ostensoir qui porte cette simple inscription † HIC EST SANGUIS XPI. Le médaillon fixé à la staurothèque de l'impératrice Marie et le petit cylindre en cristal enfermé dans un étui en cuivre sont accompagnés d'une légende métrique grecque plus élaborée mais que seules des variantes de graphie distinguent de part et d'autre. La présence de ce texte sur deux objets très dissemblables par ailleurs, et exécutés à plus d'un siècle d'intervalle, témoigne d'une tradition remarquablement vivace. Des considérations du même ordre ont dû présider au choix de l'ampoule fatimide en guise de récipient du sang du Christ. Le galbe de ce petit vase n'est pas sans rappeler l'étui métallique destiné à enchâsser le cristal byzantin correspondant et dont le couvercle est surmonté d'un second récipient plus petit, de forme bombée. Il y a tout lieu de supposer que le rapprochement avec le Saint-Sépulcre, déjà signalé dans le second cas, était aussi valable dans le premier. La similitude extérieure des deux reliquaires est donc fonction de la nature similaire de leur contenu. Jamais œuvre d'art musulman n'a été convertie de façon plus heureuse en objet de culte chrétien.

L'examen de divers autres reliquaires de Saint-Marc fera apprécier, de son côté, l'attitude des orfèvres occidentaux vis-à-vis des modèles apportés de Byzance. On observera un attachement réfléchi mais nullement systématique. L'intérêt est surtout marqué en ce qui concerne l'arrangement et les dispositions générales. Le style n'était pas pris en considération: peut-être y attachait-on moins d'importance.

La staurothèque de l'impératrice Marie restaurée en 1517, constitue apparemment un exemple de simple copie, à la fois fidèle et inexacte. Le restaurateur a scrupuleusement reproduit la dédicace de l'original grec, sans doute en est-il de même de l'iconographie, mais la facture n'a 
rien de byzantin, et c'est une œuvre du baroque tardif que nous avons à connaître, en définitive.

Des considérations de cet ordre prennent une valeur particulière dans un autre cas. Nous avons en vue un tableau en argent doré, orné de motifs végétaux et de têtes d'angelots qui encadrent un alvéole destiné à enchâsser l'un des clous de la Passion ${ }^{1}$. Il eut été tentant de supposer qu'un artiste vénitien du XVIIIe siècle avait voulu imiter ainsi une straurothèque byzantine en forme de tableau, comme le trésor de SaintMarc lui en proposait plusieurs exemples. Il suffisait de remplacer la relique de la Vraie Croix par celle du Clou. C'eut été cependant adapter le modèle à un usage nouveau. Rien n'est moins certain. La relique du Clou, déposée à une date indéterminée au trésor de la basilique, demeura cachée pendant un certain temps pour ne reparaître qu'en 1468: elle était enchâssée alors dans un étui d'origine byzantine en forme d'icône ${ }^{2}$. L'objet n'existe plus; la conclusion ne s'en impose pas moins que l'œuvre conservée actuellement reproduit les dispositions générales de cet original disparu qui répondait à la même destination. Un reliquaire de la pierre du Saint-Sépulcre qui appartient au Musée du Louvre fait connaitre, du reste, un tableau byzantin en métal, pareillement prévue pour contenir des souvenirs de la Passion différents de la Vraie Croix ${ }^{3}$. L'icôneétui d'une époque tardive conservée à Saint-Marc laisse entrevoir l'existence d'autres monuments du même genre, d'une forme plus ou moins apparentée.

On est amené ainsi à faire confiance, - du moins en dehors du style, - à la fidélité des copistes italiens. La constatation présente un intérêt certain. Ce sera l'occasion de faire connaître un objet byzantin dont le trésor de Saint-Marc ne conserve aujourd'hui que quelques fragments détachés, et dont l'existence même aurait pu échapper dans d'autres circonstances.

La démonstration a pour point de départ l'examen de quatre pe-

1. Ibidem, p. 33, pl. XXVI, 31 .

2. R i a n t, Exuviae, II, pp. 169 et 267.

3. B. M on tes qui o u - F e z e n s c, Le reliquaire de la “Pierre du Sépulcre» de la Sainte-Chapelle du Palais, Monuments... Piot, XXXII, 1932, pl. VIIIIX. Cf. les tableaux avec des reliques différentes de la Vraie Croix enchâssées dans l'encadrement: par ex., G. S chlu m b e r g e r, Un tableau reliquaire inédit du Xe siècle, Monuments ... Piot, I, 1894, pl. XIII-XIV; Oesterreichische Kunsttopographie, VII, Stift Nonnberg in Salzburg, Vienne, 1911, pl. XV-XVIII (monuments analogues d'origine vénitienne). 
tites boîtes de forme et de dimensions presque identiques ${ }^{1}$. Chacune est fermée par un couvercle à coulisse avec une inscription niellée désignant respectivement les reliques des langes du Christ (ảjò $\tau \tilde{\omega} \nu$ á $\gamma i \omega v$

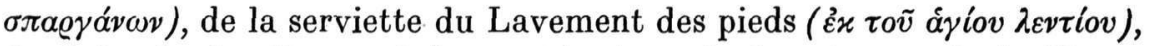
du Saint-Suaire ( $\dot{\varepsilon} x \tau \tilde{\eta} \varsigma$ áyía $\sigma v v \delta o ́ v o \varsigma)$ et de la ceinture de la Vierge

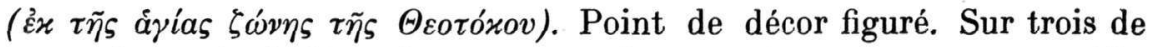
ces petits étuis, la légende est en partie masquée par un anneau mobile fixé au milieu du couvercle. La boîte qui contient la relique des langes du Christ, est un peu plus petite que les autres et n'a pas d'anneau. Il n'est pas douteux cependant que les quatre pièces faisaient partie d'un seul ensemble.

La comparaison avec une staurothèque vénitienne de la fin du Moyen Age, qui appartient également au trésor de Saint-Marc, nous permettra de reconstituer cet ensemble. L'objet, qui est relativement bien conservé, affecte la forme d'un tableau rectangulaire divisé en quatre panneaux par une rainure cruciforme garnie de parcelles de la Vraie Croix ${ }^{2}$. Le champ entre les branches de la croix est constitué par vingt cellules, dont douze ont conservé leur couvercle muni d'un anneau et de la désignation de diverses reliques. Il semble qu'il faille attribuer une destination analogue aux quatre petites boîtes accompagnées d'inscriptions grecques que l'on vient de décrire, et qui elles aussi pouvaient venir s'insérer entre les branches d'une croix, dans une staurothèque en forme de tableau, aujourd'hui disparue. De part et d'autre, le contenu est, du reste, à peu de choses près le même. Trois des cellules du tableau italien sont garnies de reliques du Précurseur et de saint Paul, les neuf autres sont réservées à des souvenirs du Christ et de la Vierge.

Plusieurs autres monuments peuvent être cités, de leur côté, à titre de comparaison, d'un caractère moins probant, il est vrai. Des dispositions plus ou moins semblables distinguent une staurothèque byzantine que le doge Morozini aurait apportée de Constantinople, et qui appartient actuellement à la collection Stoclet à Bruxelles ${ }^{3}$. Là encore, on est en présence d'un tableau divisé par une grande croix en quatre panneaux, dont chacun aurait pu être remplacé, - mais ce n'est pas le cas, - par un

1. P a s in i, Il Tesoro, p. 34 sq.; Th i e p o l o, Trattato, pp. 74, 75, 77 (l'auteur signale une cinquième boîte apparemment de la même forme, que Pasini ne connaissait déjà plus, et qui contenait une relique du vêtement de pourpre du Christ:

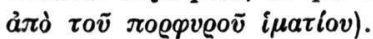

2. $\mathrm{P}$ a s in i, Il Tesoro, p. 35 .

3. Frol ow, La Relique, p. 290, no 261. 
reliquaire complémentaire. Ailleurs, comme par exemple dans les staurothèques conservées à Mettlach ${ }^{1}$, à Trèves ${ }^{2}$, à Donauwörth ${ }^{3}$, au couvent de Saints-Cyr-et-Julitte en Svanétie ${ }^{4}$ et à Vatopédi au Mont-Athos ${ }^{5}$, le champ est garni de compartiments à reliques, qui sont toutefois généralement beaucoup plus petits et soit entièrement scellés, soit fermés par de minuscules portes à gonds qui s'ouvrent mais ne s'enlèvent pas ${ }^{6}$.

La ressemblance devait être plus accusée, à ces divers égards, entre la staurothèque vénitienne d'une époque tardive qui appartient au trésor de Saint-Marc et le reliquaire grec disparu, dont la basilique ne conserve aujourd'hui que quelques pièces détachées. Il est difficile de ne pas admettre que l'orfèvre italien se soit inspiré d'un modèle byzantin encore intact qu'il avait sous les yeux. Aucun des autres monuments de comparaison que l'on vient de passer en revue n'eut suffi à reconstituer celui-ci. C'est bien à cette copie que nous devons de pouvoir nous représenter l'original disparu. L'inventaire archéologique de Byzance se trouve ainsi enrichi d'une œuvre nouvelle. Rien ne peut mieux faire apprécier, en même temps, l'emprise et l'efficacité des influences byzantines sur l'art de l'Occident.

\section{A. FROLOW}

1. Ibidem, p. $412, \mathrm{n}^{\circ} 503$.

2. Ibidem, p. $413, \mathrm{n}^{\circ} 504$.

3. Ibidem, p. 268, no 206.

4. Ibidem, p. $484, \mathrm{n}^{\circ} 662$.

5. Ibidem, p. $571, \mathrm{n}^{\circ} 886$. Cf. nos $35,464,1,467,473,494,496,591$, etc.

6. Les auteurs des descriptions anciennes appellent ces compartiments 0 ńr (ibidem, nos 332, 1, 381), cellulae ou cabinets (ibidem, nos 464, 1, 467). 